\title{
MODELO DE EQUIPO DE PRENSADO TIPO PALANCA, PARA MEJORAR LA PRODUCCIÓN DE QUESO EN LA PROVINCIA DE OCAÑA
}

\section{LEVER TYPE PRESSING EQUIPMENT MODEL TO IMPROVE CHEESE PRODUCTION IN THE PROVINCE OF OCAÑA}

\author{
MSc. Eder Flórez Solano, MSc(c). Ricardo Andrés García León \\ Ing. Marlon Escobar macea. \\ Universidad Francisco de Paula Santander Ocaña. Facultad de Ingenierías \\ Grupo de Investigación Ingap. \\ Vía Acolsure, Sede el Algodonal - Ocaña, Norte de Santander, Colombia. \\ Tel.: (+577) 5690088 Ext. 212 - 191. \\ E-mail: (enflorez, ragarcial, mrescobarm)@ufpso.edu.co.
}

\begin{abstract}
Resumen: Este trabajo presenta una alternativa para mejorar esta etapa del proceso de elaboración de queso para los pequeños y medianos productores, mediante la propuesta de un modelo de prensadora tipo palanca neumática, esta máquina que da forma y la compactación adecuada, que además, de optimizar el sistema de moldeo, tiene como finalidad principal mejorar la calidad del producto.
\end{abstract}

Palabras clave: Maquina, lácteos, prensado, diseño mecánico, calidad.

Abstract: This work presents an alternative to improve this stage of cheese processing for small and medium producers, by means of the proposal of a model of pneumatic lever type presser, this machine that gives form and the adequate compaction, that also, of optimize the molding system, its main purpose is to improve the quality of the product.

Keywords: Machine, dairy, pressing, mechanical design, quality.

\section{INTRODUCCIÓN}

El queso es uno de los derivados de la leche, debido a que aporta vitamina $\mathrm{A}$ y $\mathrm{D}$, como algunas sales minerales (Calcio, fosforo y hierro), proteínas y líquidos, además el queso se procesa en grandes cantidades tanto a nivel nacional, como internacional, y es muy asequible debido a su precio, siendo este un producto de gran presencia en la canasta familiar (Escobar Macea \& Casadiegos Noriega, 2016)

Actualmente en la provincia de Ocaña y sur del Cesar se produce distinto productos lácteos, dentro de los cuales se presenta el queso, que dentro de la jerga popular, se conoce como queso costeño, y este se produce de manera muy rudimentaria en pequeñas y medianas empresas, es necesario las comunicaciones entre las PYMES (Duran \& Iturriago., 2012).

Las empresas de la región desean aumentar la producción de queso, por lo anterior se requiere mejorar el prensado, que es el último proceso en la producción y es de vital importancia, ya que, un buen proceso asegura una mejor calidad en la contextura, y durabilidad.

Mediante visitas a diferentes empresas, y al realizar prácticas de procesamiento del queso, donde se incluía el prensado, se determinó una falencia en este último proceso. El problema de prensado se origina debido a la forma como se hace, y es de forma artesanal. 
El presente documento muestra el diseño y cálculo de una estructura de prensado, mostrando a su vez una propuesta de prensado, utilizando el principio de palanca, beneficiándonos de la ventaja mecánica que esta genera, para así mejorar el prensado del queso y obtener una mejor producción y un mejor producto.

\section{DESARROLLO DEL PROYECTO}

La producción de queso costeño consta de los siguientes pasos:

- Recolección de la leche.

- Pasteurización

- Adición del cuajo

- Salado

- Moldeado

- Prensado

Por medio de los pasos anteriores se obtiene el producto final de Queso costeño, tras el análisis de estas etapas se observó que actualmente el proceso de prensado era rustico y no proporcionaba las presiones adecuadas que se debían aplicar a la cuajada para lograr una correcta compactación del producto.

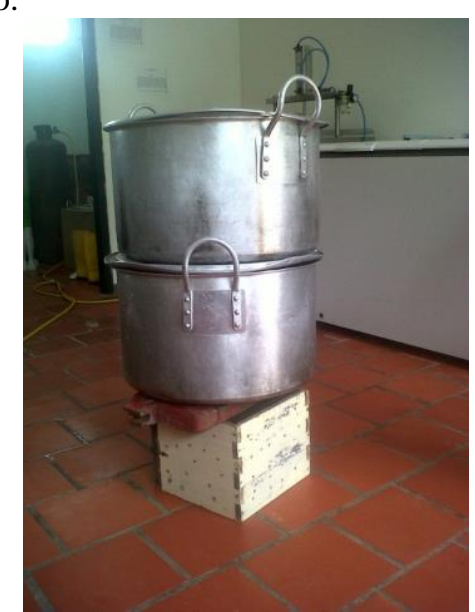

Fig. 1. Prensado artesanal Fuente: Autores.

Por esto se decidió enfocarse en el desarrollo de un tipo de prensa que además de ser apta para el proceso de prensado la economía de su construcción la situara dentro de una opción viable para los productores.

Se requiere mejorar el último paso de la producción del queso costeño, y es el prensado; basándonos en las recomendaciones de la cartilla (Manual De Lácteos Del Sena, 2010). Utilizadas en el las empresa de lácteos, para la obtención de un buen producto, y mejorar la calidad del producto.

Ya que actualmente en las prensas comercializadas son de tipo horizontal o vertical ninguna de esta utiliza el efecto palanca y por esto se plantea el diseño de una prensa tipo palanca con el fin de obtener un aumento de fuerzas a bajo costo.

Para cumplir con esto se plantea hacer un prototipo de prensa neumática y acero inoxidable con el cual se asegura no tener derrames de fluidos y partículas que atenten contra la calidad del producto, además de un sistema de palanca con el cual es posible equilibrar una resistencia grande, prensar intensamente un queso con una pequeña potencia y escaso peso siempre que esta se coloque de modo que el brazo de la palanca sea bastante mayor que la longitud del brazo resistente" (Matallana Ventura, Santiago. 1992) (Maldonado., L. C. Tarantino A. R. Sandra Aranguren., S. Peñaloza S., S. 2009).

Tras la realización de pruebas de ensayo de prensado y determinar la relación entre el peso del queso y la fuerza aplicada a este:

$R_{f}=\frac{w_{\text {Total }}}{w_{\text {cuajada }}}$

Y se encontró que en el proceso de prensado normalmente realizado se obtenía una relación de:

$R_{f}=5,3155$

Por lo anterior, como no se obtenía un producto con las condiciones de dureza adecuada, se decidió aumentar la fuerza con un sistema de palanca el cual lograba triplicar la fuerza aplicada sobre el queso llegando a la siguiente relación:

$R_{f}=11,14$

Diseño y cálculo del equipo de prensado

Con las áreas transversales que se tienen disponibles para el diseño son las siguientes:

Tubería cuadrada de $3 / 4$ pulgada, de 1 pulgada, y de 2 pulgada; con los siguientes calibres 16 y 18 . 


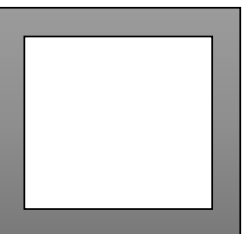

Fig. 2. Sección transversal de tubería cuadrada Fuente: Autores.

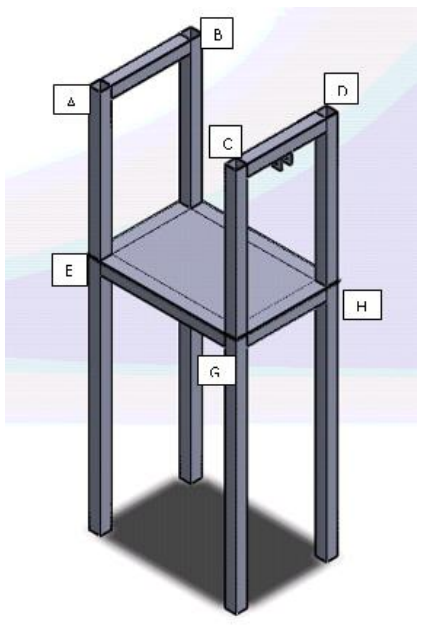

Fig. 3. Estructura de la mesa de prensado Fuente: Autores.

2.1 Diagrama de palanca I: Se seleccionó un acero inoxidable 304 , con las siguientes propiedades:

Módulo de elasticidad (E): 190,0 GPa.

Resistencia a la fluencia $\left(\boldsymbol{S}_{\boldsymbol{y}}\right): 276 \mathrm{MPa}$.

Módulo de rigidez (G): $73 \mathrm{GPa}$

Para la solución estática del diagrama de la barra, se trabajó como una viga simple, apoyada en el punto extremo izquierdo de la carga del actuador, a $20 \mathrm{~cm}$ de la fuerza aplicada sobre la cuajada.

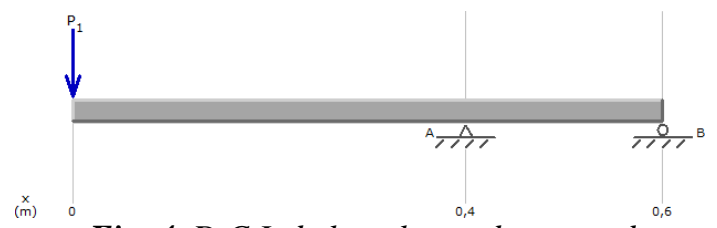

Fig. 4. D.C.L de la palanca de prensado.

Fuente: Autores.

2.2 Fuerzas $y$ reacciones presentes en la palanca:

$F_{A_{y}}=2436,25 \mathrm{~N}$

$F_{A_{x}}=532,06 \mathrm{~N}$

$R_{Q_{x}}=7307,47 \mathrm{~N}$
$R_{Q_{y}}=1889,84 \mathrm{~N}$

$O_{x}=2421,56 \mathrm{~N}$

$O_{y}=4871,22 \mathrm{~N}$

La palanca se encarga de triplicar la fuerza generada por el actuador para trasmitirla a la barra de prensado de queso.

Para el diseño de la palanca analizaremos los esfuerzos máximos tanto esfuerzo a flexión $(\sigma)$ para compararlo con el esfuerzo máximo del cortante $(\tau)$ y ver su incidencia.

Esfuerzo a flexión máximo:

$\sigma_{\max }=\frac{M c}{I}$

Donde:

M: Momento máximo presente en el diagrama de momento flector de la palanca.

C: Distancia del eje neutro a la fibra o punto a analizar.

I: Momento de inercia del área transversal de la palanca, en este caso un círculo.

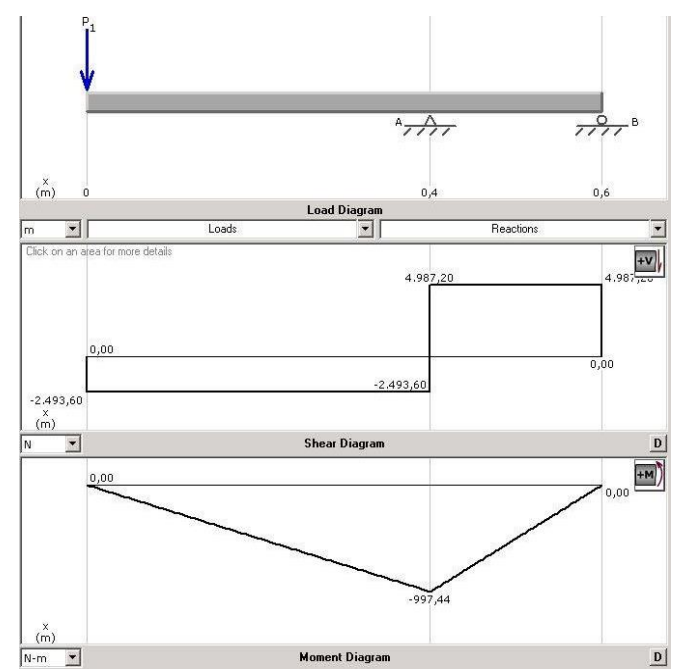

Fig. 5. Diagrama de cortante y momento flector de la palanca

Fuente: Autores del proyecto.

Dimensiones del área transversal del tubo circular macizo:

Diámetro: $40 \mathrm{~mm}$

Distancia C: $20 \mathrm{~mm}$

Inercia:

$I=\frac{\pi(0,04)^{4}}{64}$ 
$I=1,2566 \times 10^{-7} \mathrm{~m}^{4}$

$\sigma_{\max }=\frac{997,44(0,02 \mathrm{~m})}{1,2566 \times 10^{-7} \mathrm{~m}^{4}}$

$\sigma_{\max }=158,75 \mathrm{MPa}$

\subsection{Esfuerzo cortante máximo:}

$\tau_{\max }=\frac{V Q}{I t}$

Donde:

V: Cortante máximo presente el diagrama de cortante.

Q: Producto de área (A) por $\bar{Y}$

t: Espesor de la fibra o tramo a analizar.

$Q=\frac{\pi r^{2}}{4} \frac{4 r}{3 \pi}$

$Q=\frac{\pi(0,02 m)^{2}}{4} \frac{4(0,02 m)}{3 \pi}$

$Q=2,666 \times 10^{-6} \mathrm{~m}^{3}$

$\tau_{\max }=\frac{4987,2 \mathrm{~N}\left(2,666 \times 10^{-6} \mathrm{~m}^{3}\right)}{1,2566 \times 10^{-7} \mathrm{~m}^{4}(0,04 \mathrm{~m})}$

$\tau_{\max }=2,64 M P a$

Comprando los resultados de los esfuerzos cortante máximo y flector máximo llegamos a la conclusión de que solo se tendrá en cuenta el esfuerzo flector máximo presente en la palanca ya que el esfuerzo cortante máximo es muy pequeño e insignificante respecto al esfuerzo flector máximo (García-León, Flórez Solano, \& Acosta Pérez, 2015).

\subsection{Factor de seguridad}

$n=\frac{S y}{\sigma_{\max }}$

$n=\frac{276 M p a}{158,75 M P a}$

$n=1,74$

Con un factor de seguridad de 1,74 podemos confiar en que una palanca de diámetro de $40 \mathrm{~mm}$ y longitud estimada de $600 \mathrm{~mm}$ soportara las cargas a las que estará sometido.

Para el diseño de esta barra que realiza el proceso de transferir la fuerza de la palanca al molde para compactar la cuajada se procederá a determinar en primero a hacer el respectivo análisis de columna.

Determinamos la relación de esbeltez para saber qué tipo de análisis se le deberá realizar. Con el anterior análisis geométrico sabemos que la longitud de la barra debe ser de 0.3 metros.

\section{L: 0.3 metros}

Del diseño anterior sabemos que los valores de K, E, Sy y A para dimensiones de tubería cuadrada de 2 " de calibre 18 son:

K: $0.02024 m$

E: $190 \mathrm{MPa}$

Sy:276 $M P a$

A: $2,41 \times 10^{-4} m^{2}$

$\left(\frac{l}{k}\right)_{1}=\left(\frac{2 \times \pi^{2} \times C \times E}{S_{y}}\right)^{1 / 2}$

$C=1$ Para la condición de articulado en ambos extremos para análisis de columnas.

$\left(\frac{l}{k}\right)_{1}=116,5$

Evaluando el 1/k y comparamos con $\left(\frac{l}{k}\right)_{1}$.

$\frac{l}{K}=\frac{0,3}{0,02024}$

$\frac{l}{K}=914,8$

Ya que el $L / K \ll\left(\frac{L}{K}\right)_{1}$ entonces no se aplica columnas y analizamos por compresión pura.

$\mathrm{F}=547,89$ Newton

$A=2,41 \times 10^{-4} \mathrm{~m}^{2}$

Compresión pura:

$\sigma=\frac{F}{A}$

$\sigma=\frac{7547,89 \mathrm{~N}}{2,41 \times 10^{-4} \mathrm{~m}^{2}}$ 
$\sigma=31,32 \mathrm{MPa}$

Factor de seguridad (n):

$n=\frac{276 \mathrm{MPa}}{31,32 \mathrm{MPa}}$

$n=8,8$

El valor de 8,8 que nos proporciona el factor de seguridad nos indica que se puede usar para las patas de la mesa una tubería cuadrada de 2 "x2" de calibre 18 .

\subsection{DISEÑO DE UNIONES Y SUJECIONES}

Diseño de pasadores: Los pasadores se han diseñado teniendo en cuenta la mayor fuerza que se aplica en la palanca, la cual se encuentre en el punto "o" (ver imagen de la palanca).

\section{Fuerza total:}

$F_{\text {Total }}=\sqrt{R_{Q y}{ }^{2}+R_{Q x}{ }^{2}}$

$F_{\text {Total }}=\sqrt{(7307,47 N)^{2}+(1889,84 N)^{2}}$

$F_{\text {Total }}=7547,8879 \mathrm{~N}$

Encontrar el área de pasador:

$\tau=\frac{F}{A}$

Donde:

F: fuerza que soporta el pasador.

A: área transversal donde se concentra la fuerza.

$276 \mathrm{MPa}=\frac{7547,8879 \mathrm{~N}}{A}$

Despejando "A" de la ecuación, se obtiene:

$A=2,7347 \times 10^{-5} \mathrm{~m}^{2}$

"A" corresponde al área transversal del pasador, ahora se encuentra su diámetro:

$A=\frac{\pi}{4} D^{2}$
$2,7347 \times 10^{-5}=\frac{\pi}{4} D^{2}$

Despejando “D”, se tiene:

$D=5,90 \times 10^{-3} \mathrm{~m}$

Recomendación del pasador (poner referencia)

$\emptyset_{\text {Pasador }}>5,90 \times 10^{-3} \mathrm{~m}$

Se pasa al siguiente diámetro, es decir, se escoge uno a mayor al diámetro que se tiene, pero que a su vez este sea comercial, para este caso, se selecciona un diámetro para el pasador de $8 \mathrm{~mm}$. Entonces se encuentra el cortante que soporta el pasador, con el diámetro seleccionado. (Budynas, R. 2012)

$\tau=\frac{7547,8879 N}{\left(\frac{\pi}{4}\right) \times\left(8 \times 10^{-3}\right)^{2}}$

$\tau=150,160 \mathrm{MPa}$

Para el factor de seguridad del pasador:

$n=\frac{276 M P a}{150,160 M P a}$

$n=1,838$

2.6 Análisis de esfuerzo en los soportes de la palanca: El esfuerzo esta dado de la siguiente manera:

$\sigma=k_{t} \frac{F}{A}$

Donde:

F: fuerza que soporta el soporte.

A: área transversal del soporte.

$\boldsymbol{k}_{\boldsymbol{t}}$ : Factor de concentrador.

Tomando el área recomendada se tiene:

$A=(w-d) \times t$

Donde:

W: es el ancho del soporte.

D: diámetro del pasador.

T: el espesor del soporte. 
H: altura del soporte.

A continuación se muestra las distintas dimensiones, para cada parámetro establecido para encontrar el área, y el concentrador de esfuerzo.

$d=8 \mathrm{~mm}, w=40 \mathrm{~mm}, h=20 \mathrm{~mm}$

$\frac{h}{w}=\frac{20}{20}$

$\underline{h}=1$

$\bar{w}=1$

$\frac{d}{w}=\frac{8}{40}$

$\frac{d}{w}=0,2$

$k_{t}=5,4$

Se seleccionó un concentrador de esfuerzo $k_{t} 5,4$. Para una fuerza de $5439,92 \mathrm{~N}$, se divide entre el número de soportes, pues esta se distribuye de igual forma en cada uno de los soportes, entonces se tiene:

$F=\frac{5439,92 N}{2}$

$F=2719,96 N$

Si se varía el espesor de cada placa, cambia su área y también su esfuerzo, analizaremos como es el cambio en los siguientes espesores:

$\mathrm{t}=3 \mathrm{~mm}$

$\sigma=(5,4) \times \frac{2719,96}{9,6 \times 10^{-5}}$

$n=1,35$ Calibre 8

$\sigma=153 \mathrm{MPa}$

$\mathrm{t}=4,16 \mathrm{~mm}$

$\sigma=(5,4) \times \frac{2719,96 \mathrm{~N}}{1,33 \times 10^{-4} \mathrm{~m}^{2}}$

$n=1,88$ Calibre 8

$\sigma=110,43 \mathrm{MPa}$

$\mathrm{t}=4,58 \mathrm{~mm}$ $\sigma=(5,4) \times \frac{2719,96 \mathrm{~N}}{1,6 \times 10^{-4} \mathrm{~m}^{2}}$

$n=2,27$ Calibre 6

$\sigma=91,8 \mathrm{MPa}$

Verificando la incidencia del esfuerzo cortante directo presente en el soporte tenemos para calibre 8:

$\tau=\frac{F}{A}$

$\tau=\frac{2421,56 \mathrm{~N}}{1,33 \times 10^{-4} \mathrm{~m}^{2}}$

$\tau=18,21 \mathrm{MPa}$

Esfuerzo de Von Mises:

$$
\begin{aligned}
\sigma^{\prime} & =\sqrt{\sigma_{x}^{2}+3 \tau_{x y}^{2}} \\
\sigma^{\prime} & =\sqrt{(110,43 M P a)^{2}+3(18,19 M P a)^{2}} \\
\sigma^{\prime} & =123,18 M P a \quad \therefore n=2,24
\end{aligned}
$$

Como resultado se puede trabajar con cualquier espesor superior al calibre 8 para el diseño del soporte, ya que nos asegura un factor de seguridad de 2,24 lo cual indica confiabilidad del diseño.

2.7 Análisis de esfuerzo en los soportes barra de prensado: Dimensiones asumidas para la parte en contacto con la palanca:

$d=8 \mathrm{~mm}$

$w=5 \mathrm{~cm}$

$h=2,5 \mathrm{~cm}$

$\sigma=k_{t} \frac{F}{A}$

Se divide la fuerza para los dos soportes por igual.

$F_{\text {soporte }}=\frac{7547,89 \mathrm{~N}}{2}$

$F_{\text {soporte }}=3773,9 \mathrm{~N}$

$k_{t}$ Se halla en la tabla A-15-12. (Budynas, R. 2012)

$A=(w-d) \times t$ 


$$
\begin{aligned}
& \frac{d}{w}=\frac{0,8 \mathrm{~cm}}{5 \mathrm{~cm}} \\
& \frac{d}{w}=0,16 \\
& \frac{h}{w}=\frac{2,5 \mathrm{~cm}}{5 \mathrm{~cm}} \\
& \frac{h}{w}=0,5 \\
& k_{t}=6,23
\end{aligned}
$$

Realizando el procedimiento para los espesores $(t)$ de $3 \mathrm{~mm}$ y $4 \mathrm{~mm}$ usando $\mathrm{Sy}=276 \mathrm{MPa}$

Para:

$t=3 m m \sigma=186,59 \mathrm{MPa} \mathrm{Y}$ un $n=1,47$

$t=3,5 \mathrm{~mm} \sigma=159,9 \mathrm{MPa} \mathrm{Y}$ un $n=1,72$

$t=4 m m \sigma=139,94 \mathrm{MPa} \mathrm{Y}$ un $n=1,97$

Puede usarse un espesor para el soporte en contacto con la palanca con un espesor superior a $3 \mathrm{~mm}$.

2.8 Soporte del molde: Dimensiones:

$$
\begin{aligned}
& d=8 \mathrm{~mm} \\
& w=2 \mathrm{~cm} \\
& h=2 \mathrm{~cm}
\end{aligned}
$$

Para este soporte se trabaja con la fuerza completa de $7547.89 \mathrm{~N}$

$$
\begin{aligned}
\sigma & =k_{t} \frac{F}{A} \\
A & =(w-d) \times t \\
\frac{d}{w} & =\frac{0,8 \mathrm{~cm}}{2 \mathrm{~cm}} \\
\frac{d}{w} & =0,4 ; \frac{h}{w}=\frac{2 \mathrm{~cm}}{2 \mathrm{~cm}} ; \frac{h}{w}=1 \\
k_{t} & =6,23
\end{aligned}
$$

Para espesores $(t)$ de 8 a $10 \mathrm{~mm}$

$\begin{array}{ll}t=10 m m \sigma=138,37 M P a \mathrm{Y} \text { un } & n=1,9 \\ t=8 \mathrm{~mm} \sigma=172,97 \mathrm{MPa} \mathrm{Y} \text { un } & n=1,59\end{array}$

Se puede trabajar con un soporte de espesor $(t)$ superior a $8 \mathrm{~m}$.

\subsection{Diseño de uniones permanentes (soldadura)}

Los esfuerzos en uniones soldadas sujetas a flexión, se determinan con el cortante nominal que se presenta en la garganta, y está dada por la siguiente formula:

$\tau=\frac{M \times c}{I}=\frac{1,414 \times M}{b \times d \times h}$

Donde:

B: ancho de la tubería cuadrada.

D: altura de la tubería cuadrada.

H: garganta de la soldadura.

Se diseñó la factura con un cortante igual a 276 $\mathrm{MPa}$, que es a su vez la resistencia a la fluencia del material de toda la estructura.

Las dimensiones de trabajo, son las mismas para todo el diseño.

Hallamos "h"

$b=d=50,8 \times 10^{-3} \mathrm{~m}, M=535,68 \mathrm{Nm}, \tau=138 \mathrm{MPa}$

$138 M P a=\frac{1,414 \times(535,68 \mathrm{Nm})}{\left(50,8 \times 10^{-3} \mathrm{~m}\right)^{2} \times h}$

$h=2,127 \times 10^{-3} \mathrm{~m}$

Después de hallada la garganta de la soldadura, se encuentra su área, su $\hat{X}$ y su $\hat{Y}$ y $I_{u}$.

$$
\begin{aligned}
& A=1,414 \times h(b+d) \quad(14) \\
& \begin{array}{l}
A=1,414 \times 2,127 \times 10^{-3} \mathrm{~m}\left(50,8 \times 10^{-3} \mathrm{~m}+50,8 \times 10^{-3} \mathrm{~m}\right) \\
A=3,055 \times 10^{-4} \mathrm{~m}^{2}
\end{array} \\
& \hat{X}=\hat{Y}=\frac{b}{2}(13) \\
& \hat{X}=\hat{Y}=\frac{50,8 \times 10^{-3} \mathrm{~m}}{2} \\
& \hat{X}=\hat{Y}=25,4 \times 10^{-3} \mathrm{~m} \\
& I_{u}=\frac{d^{2}}{6} \times(3 b+d) \\
& I_{u}=\frac{\left(50,8 \times 10^{-3} \mathrm{~m}\right)^{2}}{6} \times\left(\left(3 \times 50,8 \times 10^{-3} \mathrm{~m}\right)+50,8 \times 10^{-3} \mathrm{~m}\right) \\
& I_{u}=8,739 \times 10^{-5} \mathrm{~m}^{3}
\end{aligned}
$$

Para la soldadura de la estructura se propone usar un electrodo E27610 y un ancho de filete de $3 \mathrm{~mm}$. 
Posteriormente se realizó un análisis de los elementos que mayor mente esta sometidos a cargas, Por medio de la herramienta de diseño SolidWorks se procedió a analizar cada uno de los elementos diseñados observando sus deflexiones, esfuerzos (Gómez., G, S. 2010; Moreno., C. et al. 2012; Torres et al., 2013).

Conociendo de esta manera el comportamiento de estos elementos ante las cargas establecidas con el fin de predecir posibles fallas.

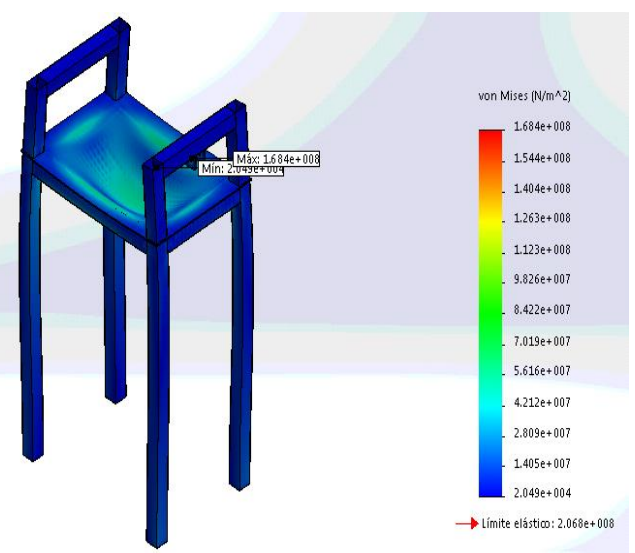

Fig. 6. Análisis de mesa de prensado esfuerzo von mises.

Fuente: Autores

Esta pieza muestra un esfuerzo máximo de $168 \mathrm{MPa}$ con el cual se asegura que el material seleccionado resistirá (Rodríguez O et al., 2012).

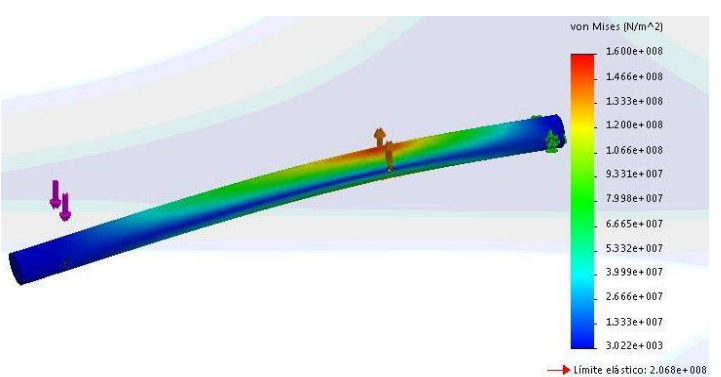

Fig. 7. Análisis palanca de prensado esfuerzo von mises.

Fuente: Autores

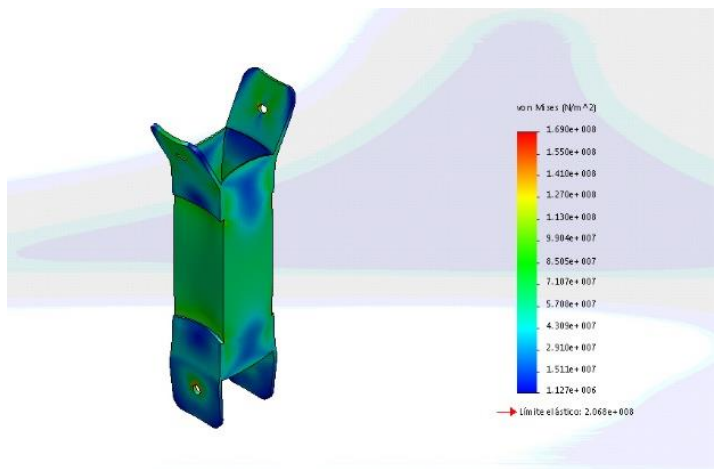

Fig. 8. Palanca de prensado. Fuente: Autores.

Tras analizar los resultados obtenidos por el software se procede a realizar la selección del sistema neumático para el accionamiento de cargas en la prensa:

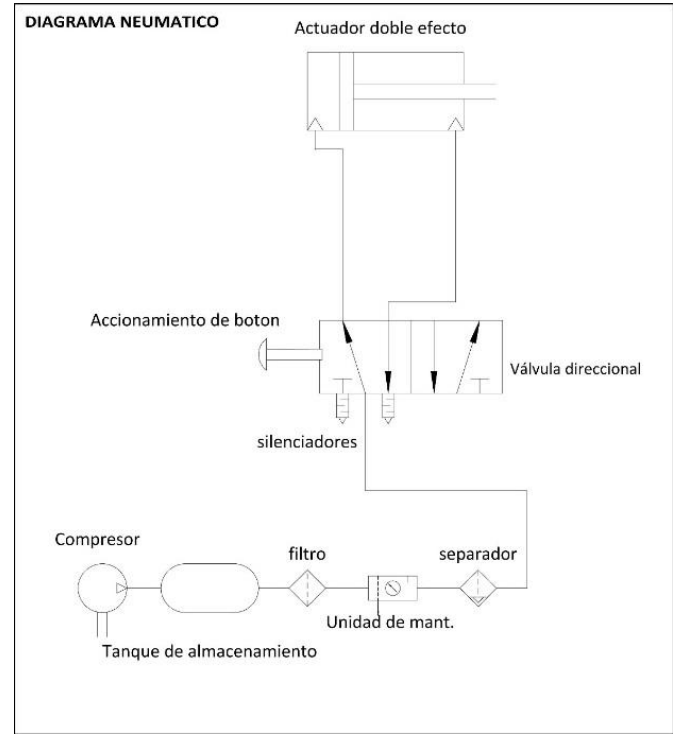

Fig. 9. Diagrama sistema neumático. Fuente: Autores.

\section{CONCLUSIONES}

La utilización de la prensa neumática ayudará a aplicar una mayor carga con el uso de menores recursos, por lo que se triplica la fuerza aplicada asegurando una mayor capacidad de compactación de la cuajada; de tal manera que se podría acortar el tiempo prensado, con lo que evidentemente se optimizará el proceso de producción del queso.

Con la prensa neumática la aplicación de la fuerza es de $52 \%$; además, se aumenta la producción del queso, debido a que el resultado final después del prensado no será de $10 \mathrm{Kg}$ sino de $35 \mathrm{Kg}$, lo cual 
repercute en un optimizar la producción en un $71 \%$.

La barra que compacta el queso es la parte de la estructura que soporta la mayor fuerza y flexión, asimismo este componente es del mismo material en toda la estructura y su geometría es circular.

La implementación de un sistema neumático facilitará la aplicación de fuerzas sobre la cuajada, y los tiempos de aplicación con la ayuda de un regulador de presión.

El uso de un sistema electrónico regulador de caudal permitirá mejorar el manejo de las presiones entrantes en el actuador debido a que este procedimiento se haría de manera automática.

\section{REFERENCIAS}

Arroyo, M. y C. García. (1988). Quesos de España. Espasa-Calpe S.A. ed. Madrid.

Bonet, B., M. Juárez, B. Moreno, R.M. Ortega, y L. Suárez. (2009). Libro Blanco de los Lácteos. Federación Nacional de Industrias Lácteas. Madrid.

Casado, P. (1991). Guía para el análisis químico de la leche y los derivados lácteos. Ayala S.A. ed. Madrid.

Castillo, M. (2001). Predicción del tiempo de corte en la elaboración de queso mediante dispersión de radiación de infrarrojo próximo. Tesis Doctoral. Universidad de Murcia. Murcia.

Chacón, A. (2006). Tecnología de membranas en la industria láctea. Agronomía Mesoamericana. 17(02):243-263.

De Alba, J. (1985). Asociación mexicana de producción animal.

Duran Ramírez, Felipe. (2009). Lácteos y Derivados - obtención-conservación-procesos.

Durán Acevedo Christian M, Iturriago Ali Xavier. (2012). Automatización de un Sistema de Suministro de Agua Potable a Través de la Tecnología Zigbee. Revista colombiana de tecnologías de Avanzada 1 (19), Pág. 36 - 42

Escobar Macea, M., \& Casadiegos Noriega, V. E. (2016). Propuesta de un modelo de equipo de prensado tipo palanca, para mejorar la producción de queso en el laboratorio de lácteos de la Universidad Francisco de Paula Santander. Norte de Santander: Universidad Francisco de Paula Santander Ocaña.

García-León, R. A., Flórez Solano, E., \& Acosta Pérez, M. A. (2015). Análisis estructural de una máquina prensadora para producción de ladrillo macizo para las pequeñas industrias artesanales de materiales cerámicos en Ocaña Norte de Santander y en la región. Revista Colombiana de Tecnologías de Avanzada, Vol.1, No 7.

Gómez., G, S. (2010). Solidworks Simulation. ALFAOMEGA. México.

Harbutt J. (1998). Productos lácteos.

Hill A. (2006). Dairy Chemistry and Physics.

Marco R. Meyer. (1988). Manual para la educación agropecuaria. Elaboración de productos lácteos. 6ta Edición.

Jeffrey Kaner Robert. (1982). Utilización de leche cruda Vs pasteurizada en la elaboración de queso.

Lácteos y Derivados. Obtención, conservación y procesos.

Libro Blanco De Los Lácteos. (2010). Libro blanco de los lácteos.

Maldonado., L. C. Tarantino A. R. Sandra Aranguren., S. Peñaloza S., S. (2009). "Ingeniería De Automatización Para El Proceso De Humectación De La Arcilla En Las Industrias Del Norte De Santander Colombia". Revista Colombiana de Tecnologías de Avanzada, Vol.1, No. 13.

Manual De Lácteos Del Sena. (2010) Derivados lácteos. Procesamiento de quesos blancos.

Manual De Práctica Para Procesar Alimentos A Base De Leche. (2009). Colegio de estudios científicos y tecnológicos, Michoacán, México.

Manuales Para Producción Agropecuaria. (2009) Elaboración de productos lácteos.

Matallana Ventura, Santiago. (1992). Prensado del queso. Sánchez, C. Producto lácteos.

Moreno., C. G. Flórez., E. Peña., C. C. (2012). Aplicación Social De La Asignatura Diseño Mecánico En La Carrera De Ingeniería Mecánica. Revista Colombiana de Tecnologías de Avanzada, Vol.2, No. 20.

SENA. (1987). Derivados Lácteos. Derivados lácteos.

Universidad Nacional. (2005). Manual de elaboración de queso costeño amasado.

Vargas Arredondo J. (2008). Manual de industrialización de la leche. ITA No. 20 Morelia, Mich.

Villega De Gante. (2004). Procesos de elaboración del queso. 2004.

Colanta, Escuela De Quesos. (2009). Historia del queso en Colombia: http://www.escueladequesos.com/el-queso/34contenidos/78-historia-del-queso-en-colombia

Universidad De La Sabana. (2005). Comunicación social y periodismo, investigación aplicada: http://www.unisabana.edu.co/carreras/comunic acion-social-y-periodismo/trabajo-de- 
grado/opciones-de-trabajo-degrado/investigacion-aplicada/

UNAD. (2010). Investigación pura, investigación aplicada:

http://datateca.unad.edu.co/contenidos/100104/ 100104_EXE/leccin_5_investigacin_pura_inve stigacin_aplicada_investigacin_profesional.htm 1

Poncelet, Enciclopedia Del Queso. (2014) Historia del queso: http://www.poncelet.es/enciclopediadel-queso/historia.html

Directo Al Paladar, El Sabor De La Vida. (2006). Historia del

http://www.directoalpaladar.com/culturagastronomica/la-historia-del-queso.
Rodríguez Oscar Oswaldo, Pineda Pinto Ronald Fernando, Cárdenas Pedro Fabián. (2012). Herramientas EJS 3D/Matlab para el control del sistema no lineal aplicado al péndulo invertido sobre carro deslizante. Revista colombiana de tecnologías de Avanzada. 1 (19). Pág. 28 - 34.

Torres Clayton José, Archila John Faber, Tronco Mário Luiz, Becker Marcelo, Viera Porto Arthur José, Tiberti Alexander José. (2013). Estudio cinemático de una plataforma robótica para agricultura. Revista colombiana de tecnologías de Avanzada, Vol. 2, No. 22, pp. 131-137 